\title{
ESPAÑA SE MONTA EN EL TREN DE ALTA VELOCIDAD
}

(SPAIN HOPS ABOARD THE HIGH SPEED TRAIN)

Manuel Pérez-Beato de Cos, Ingeniero de Caminos Director de Construcción de AVE

\section{RESUMEN}

El presente artículo trata de sintetizar, de una parte, los antecedentes históricos por los que se decide la construcción de esta línea, y de otra, los parámetros esenciales que desde el punto de vista técnico la constituyen.

\section{SUMMARY}

This article tries to synthesize the historical antecedents leading up to the decision to construct this line, on the one hand, and the basic technical parameters of which it is composed, on the other.

\section{INTRODUCCIÓN}

La línea de Alta Velocidad Madrid - Sevilla ha supuesto la construcción de un nuevo enlace ferroviario entre ambas ciudades a través de Ciudad Real y Córdoba. Su moderna concepción permite desde el 21 de abril del 92 la circulación de trenes a $250 \mathrm{~km} / \mathrm{h}$, que cubre el trayecto en menos de tres horas.

Con esta línea, España se sitúa entre los tres primeros países de Europa que comercializan la alta velocidad ferroviaria. Es el primer trayecto en el que se instala el ancho de vía europeo, con el que mejorarán considerablemente, en el futuro, las comunicaciones ferroviarias con los países de nuestro entorno.

También es la primera ejecución de lo que constituirá la Red Española de Alta Velocidad para establecer un transporte rápido y seguro en los principales corredores ferroviarios de nuestro país y sus conexiones internacionales.

La gran obra de ingeniería surge de la necesidad de superar el estrangulamiento ferroviario del paso de Despeñaperros con la vía de ancho nacional.

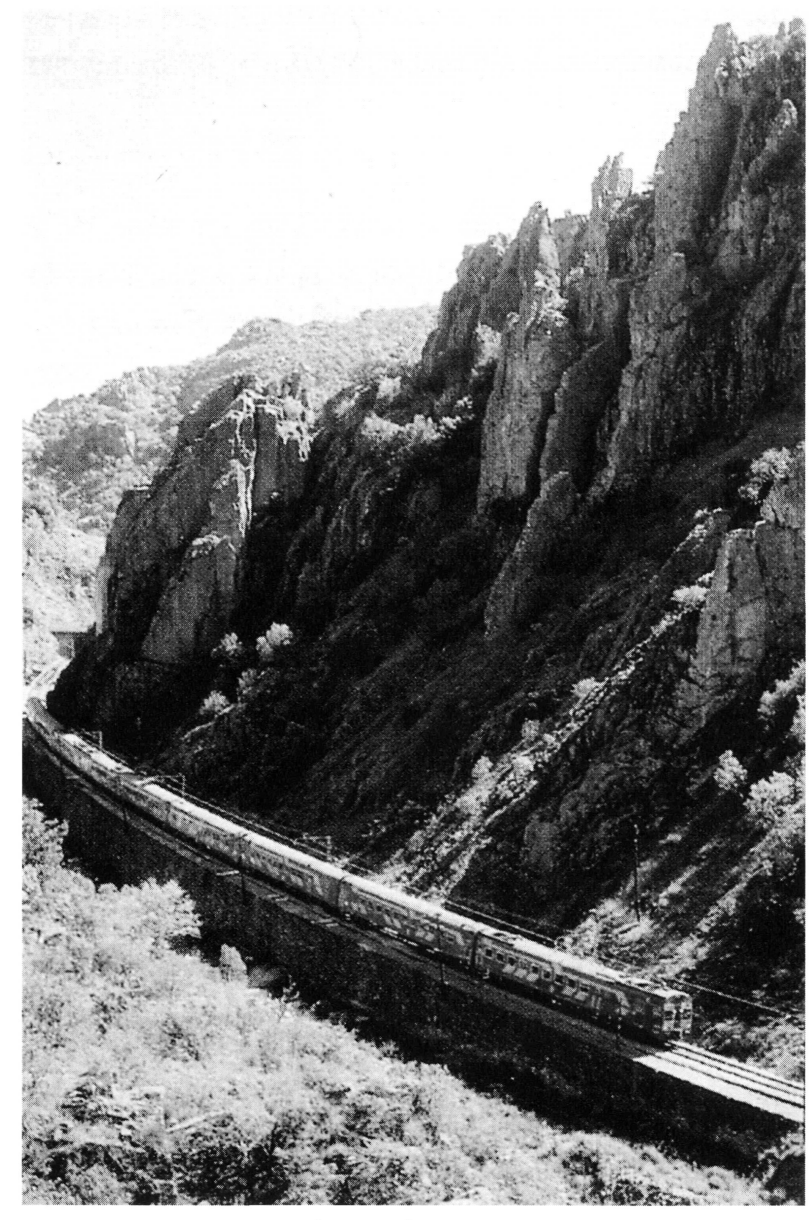

Trazado actual por Despeñaperros. 


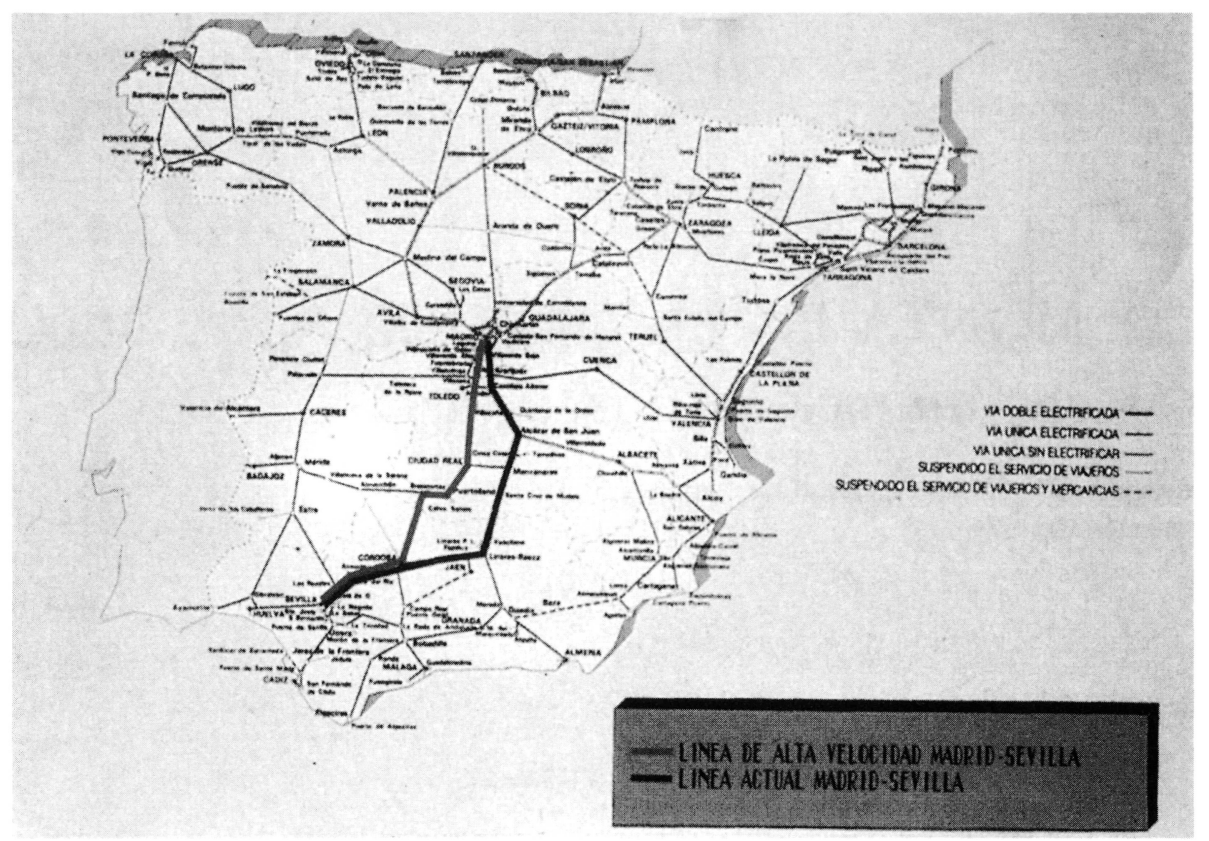

Trazado de la línea actual Madrid-Sevilla y de la línea del AVE.

La vía única del trayecto Santa Cruz de Mudela-LinaresBaeza, que conecta toda Andalucía con el resto de España, alcanza grados de saturación de capacidad tan altos que conducen a un grave deterioro de los niveles de servicio.

Para resolver la situación se estudió, en los años 1979 y 1980, la posibilidad de duplicar la vía por el trazado actual. Pero las dificultades orográficas exigían una elevada inversión con escasos resultados. Durante la construcción, además, se producirían graves interrupciones de la explotación.

Todo esto llevó a elegir la solución de construir un nuevo enlace que uniera la línea Madrid-Badajoz, a la altura de la estación de Brazatortas, con la línea MadridAndalucía, en las proximidades de Córdoba.

El 11 de octubre de 1986 el Gobierno tomó la decisión de construir el Nuevo Acceso Ferroviario a Andalucía (NAFA), que reduce la distancia entre Madrid y Sevilla en algo menos de $100 \mathrm{~km}$ y queda recogido en el Plan de Transporte Ferroviario como actuación prioritaria.

Durante el proceso de maduración de este Plan apareció en Francia el fenómeno TGV, con el inicio de la explotación de la línea París-Lyon, en 1981, que confirmó el éxito de la Alta Velocidad ferroviaria, tanto desde el punto de vista técnico como comercial. Esto avaló que la infraestructura prevista en un principio (BrazatortasCórdoba) para $160 \mathrm{~km} / \mathrm{h}$ se diseñara en el PTF y para este trazado, con los parámetros de las líneas de Alta Velocidad, aconsejando velocidades máximas no inferiores a $250 \mathrm{~km} / \mathrm{h}$. Con estas premisas se inicia en octubre de 1987 la construcción de la infraestructura ferroviaria del tramo Getafe-Córdoba $(326 \mathrm{~km})$.

El 9 de diciembre de 1988, el Consejo de Ministros toma la decisión histórica de que todas las nuevas infraestructuras ferroviarias se diseñaran para el ancho de vía UIC (1435), inferior en $233 \mathrm{~mm}$ al ancho de toda la Red Nacional de Ferrocarriles Españoles (RENFE).

Con esta decisión ya no era posible enlazar con la red existente en las proximidades de Madrid y Córdoba, por lo que fue preciso completar el proyecto existente con un acceso independiente a la nueva estación de MadridAtocha, y con la construcción de una nueva vía doble entre Córdoba y Sevilla.

Además del incremento de kilómetros de línea a realizar (143), la introducción de ancho UIC creó como dificultades inducidas la búsqueda de un trazado idóneo para penetrar a la estación de Atocha en el Centro de Madrid, y de otra el problema de suministro de materiales (carril, traviesas, balasto, etc.) desde los centros de producción a los tajos de obra. 


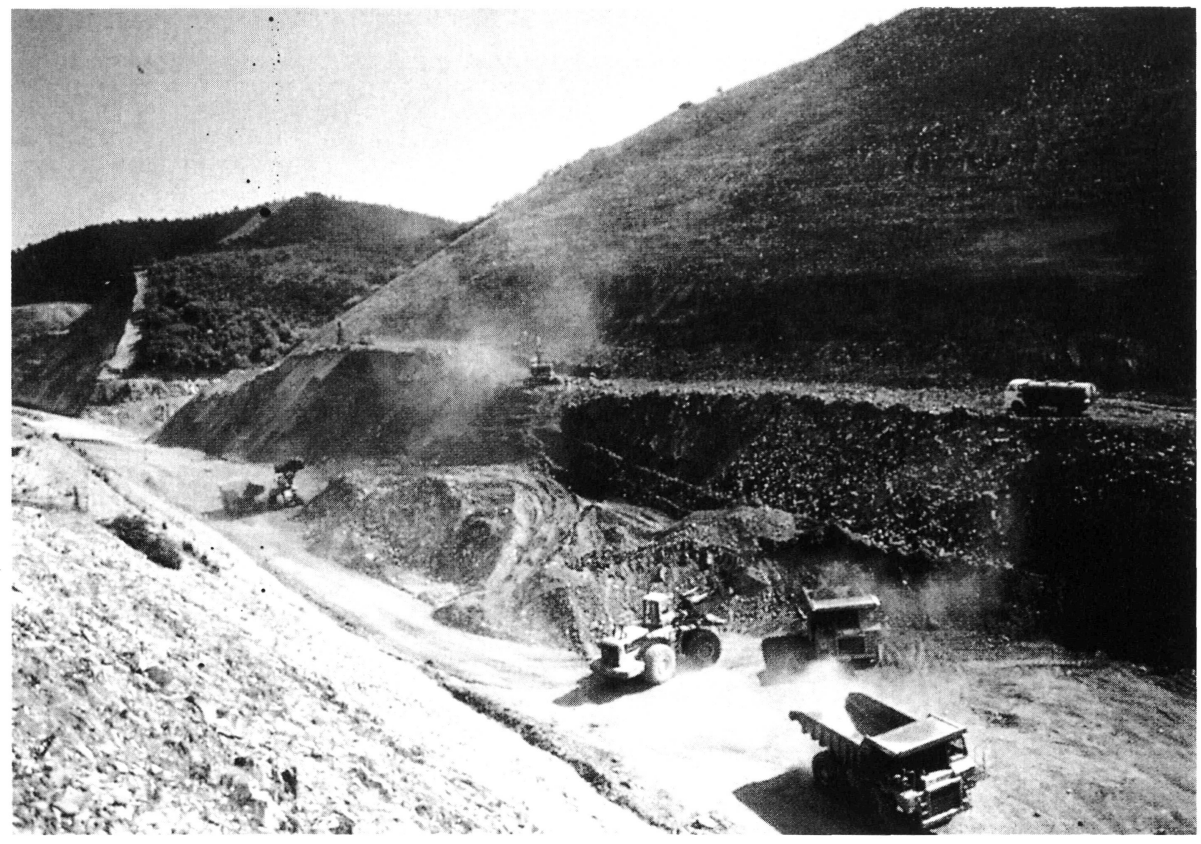

Obras de desmonte.

El PTF contemplaba como objetivo a alcanzar un tiempo de viaje entre Madrid-Sevilla inferior a tres horas. Efectuadas simulaciones de tiempo de viaje para las opciones de velocidad de $200 \mathrm{~km} / \mathrm{h}$ y $250 \mathrm{~km} / \mathrm{h}$, se comprobó que los $200 \mathrm{~km} / \mathrm{h}$ no cumplían con los objetivos marcados, por lo que se adoptó como velocidad inicial la de $250 \mathrm{~km} / \mathrm{h}$, ampliable en el futuro hasta los $300 \mathrm{~km} / \mathrm{h}$.

Respecto al tipo de tráfico, se efectuaron estudios de su distribución para aćceso a Andalucía por la nueva línea y la que está en servicio, llegándose a la conclusión de que el mejor aprovechamiento de la inversión, que disminuía considerablemente la saturación de la línea en servicio, consistía en una línea de tráfico mixto con velocidades mínimas no inferiores a $120 \mathrm{~km} / \mathrm{h}$.

\section{UNA OBRA DE GRANDES DIMENSIONES}

La nueva línea Madrid - Sevilla tiene una longitud de $471 \mathrm{~km}$. Su perfil se caracteriza por tener una primera parte (Madrid-Brazatortas) casi horizontal, con algunas excepciones, como son el paso por las depresiones de los ríos Tajo y Guadiana y el cruce de los Montes de Toledo, y una segunda parte más accidentada, en donde se suceden distintas rasantes que concluyen con un prolongado descenso hacia Córdoba, necesario para salvar una diferencia de cota de más de $600 \mathrm{~m}$ en $70 \mathrm{~km}$, lo que obliga a realizar grandes obras de infraestructura

(c) Consejo Superior de Investigaciones Científicas Licencia Creative Commons 3.0 España (by-nc) (desmontes, terraplenes, túneles, viaductos). A partir de ahí, el trazado es relativamente llano, siguiendo la cuenca del río Guadalquivir.

Las pendientes máximas son del 12,5 por mil y van enlazadas entre sí por curvas de amplios radios, para mantener elevados niveles de comodidad.

El tramo que atraviesa Sierra Morena es totalmente de nuevo trazado. En él se encuentran las obras más espectaculares, que salvan grandes accidentes naturales con un conjunto impresionante de túneles y viaductos (15 túneles y 16 viaductos).

En la construcción de la nueva línea de ferrocarril de Alta Velocidad es preciso acometer una serie de trabajos de movimientos de tierras, que permitan disponer de una plataforma de 13,30 m de ancho, con $4 \mathrm{~m}$ de sobreancho a cada lado, hasta la línea de cerramiento.

El volumen total de tierras a remover supera los $73 \mathrm{mi}-$ llones de metros cúbicos.

Los aspectos más destacados son:

- Obras de gran altura, tanto en desmontes como en terraplenes.

- Taludes tendidos.

- Medidas para protección contra desprendimientos. 
Informes de la Construcción, Vol. 44 n. ${ }^{\circ} 420$, julio/agosto, 1992

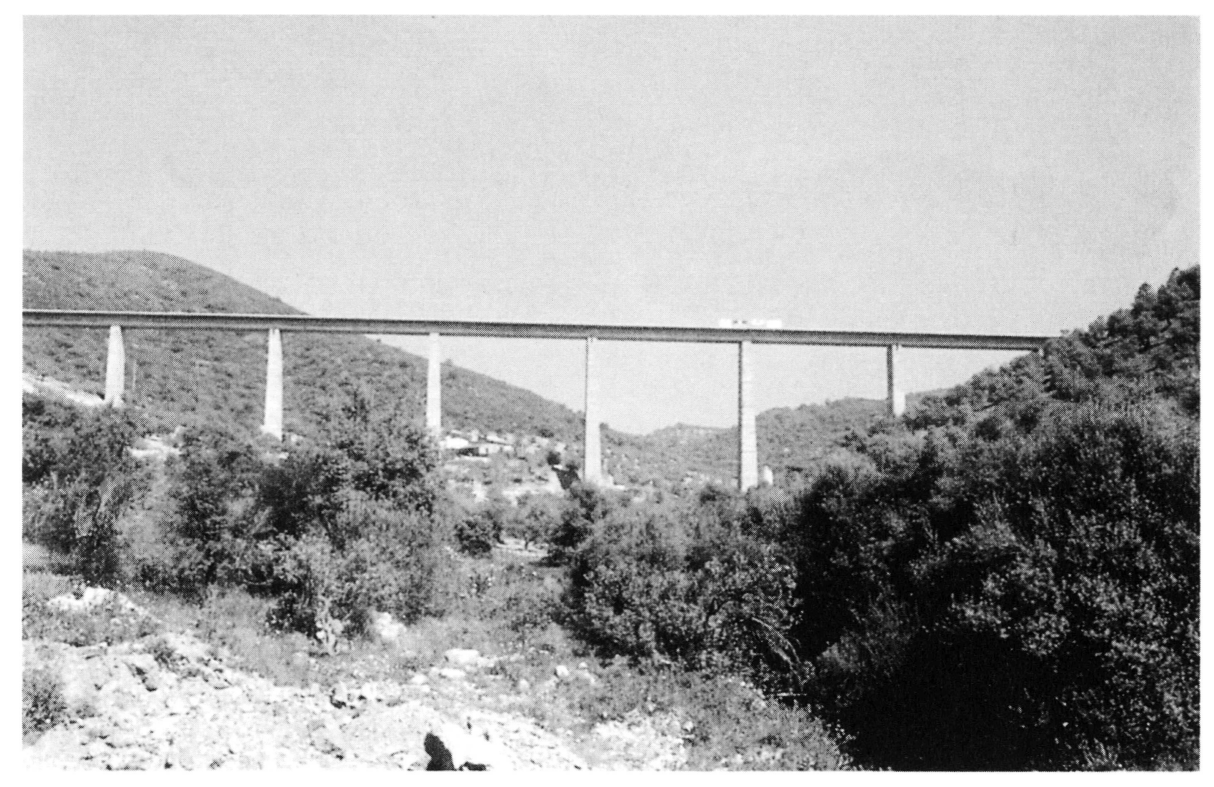

Viaducto del Concejo, en las proximidades de Adamuz (Córdoba).

- Ejecución muy cuidadosa de terraplenes de transición entre explanaciones y obras de fábrica.

- Especial cuidado en el drenaje, tanto transversal como longitudinal.

- Especificaciones estrictas de ejecución y control de calidad sobre:

- Materiales.

- Puesta en obra.

- Estabilidad de túneles.

- Compactación.

- Control y seguimiento de asientos.

La línea tiene 17 túneles, con una longitud total de $15.819 \mathrm{~m}$. El de mayor longitud tiene $2.540 \mathrm{~m}$.

La sección útil adoptada, una vez estudiados los problemas derivados de la circulación a Alta Velocidad en túnel, ha sido de $75 \mathrm{~m}^{2}$.

Esta sección, junto con la mejora introducida en los trenes de hacerlos herméticos al paso de los túneles, está demostrando su validez.

La perforación de 16 de los túneles se ha realizado mediante la técnica del "Nuevo Método Austríaco", que permite optimizar el tipo de sostenimiento, en función de las características del terreno atravesado.
El otro es un túnel artificial necesario por razones de permeabilidad.

En la nueva línea se han construido 31 viaductos, que tienen una longitud total de 9.845 metros.

Los mayores son el de la variante de Ciudad Real, de $930 \mathrm{~m}$; y los que cruzan los ríos Tajo y Guadalmez, con cerca de $800 \mathrm{~m}$. La altura máxima de pilas es de 78 metros.

La sección adoptada para los viaductos es única, y tiene una anchura total de 11,60 metros.

La tipología estructural es de 2 clases:

- Viaductos isostáticos con vanos de 26,6 a $40 \mathrm{~m}$, salvados mediante vigas prefabricadas pretensadas.

- Viaductos hiperestáticos, con vanos de hasta $50 \mathrm{~m}$, salvados mediante vigas y cajones continuos postensados.

El sistema constructivo de éstos ha sido por empuje, dovelas prefabricadas o mediante empleo de cimbras.

La vía sobre la que circulan los trenes de alta velocidad es de concepción clásica, o sea, montada en traviesas sobre balasto. Éste es enteramente de machaqueo, cumpliendo todas las normas de calidad. 
Es una vía doble, a lo largo de toda la línea, con una separación entre ejes de $4,30 \mathrm{~m}$ y un ancho de vía de $1,435 \mathrm{~m}$, que es el ancho normal europeo.

El carril utilizado es del tipo UIC-60 $(60 \mathrm{~km} / \mathrm{m})$, de mayor peso que el empleado en las líneas convencionales. Se suministra en barras de $288 \mathrm{~m}$ de longitud.

En obra, la soldadura de la barra se hace por procedimientos aluminotécnicos, para obtener una rodadura sin juntas.

Las traviesas son de hormigón postensado o pretensado, monobloque tipo DW, separadas $60 \mathrm{~cm}$ entre ejes, con sujeción Vossloh tipo HM con placa de asiento de $6 \mathrm{~mm}$ de espesor.

Apartado importante en una vía de alta velocidad lo constituyen los aparatos de vía. La explotación racional de una vía de alta velocidad exige doble vía banalizada, debiendo ser la velocidad de paso por vía desviada, en los escapes, lo más próxima posible a la de la vía directa, fijándose aquélla en el escalón superior de los recomendados por la UIC, es decir, $160 \mathrm{~km} / \mathrm{h}$. Por último, se ha considerado suficiente $80 \mathrm{~km} / \mathrm{h}$ por vía desviada para los desvíos situados en vía general, que sirven a vía de apartado. Las traviesas son de hormigón armado, debido a la mayor estabilidad que, gracias a su peso, confieren al conjunto. Los dos tipos de desvíos son el DSI-60 R 10.000-4.000 y 0,0267 de tangente y el DSI-60 R tg. 0,07 . Del primer tipo se han instalado 60 unidades y 43 del segundo.

Estos desvíos tienen un corazón móvil, disponiendo para su acoplamiento y desacoplamiento de 11 motores individuales sincronizados y dispuestos ocho en la aguja (long. 49,8 m) y tres en el cruzamiento (long. 21,7 m), habiéndose desechado la utilización de menos motores con varillaje de transmisión de esfuerzos debido a su menor fiabilidad.

Todo el montaje de vía y desvíos es importante que conserve con el tiempo, tanto en planta como en alzado, su geometría de diseño. Para ello es necesario disponer de bases de replanteo estables que bien pueden ser los postes de catenaria, ya que es suficiente disponer de puntos fijos separados $60 \mathrm{~m}$, por lo que, sobre estos postes, se ubican los puntos de replanteo. Establecidas las cuerdas y flechas correspondientes sobre estos puntos de replanteo, se ha fijado la posición absoluta de la vía, que se recoge en las hojas kilométricas de la línea, permitiendo en todo momento conocer la situación absoluta de la vía, sus variaciones y comprobar si está o no dentro de las tolerancias fijadas.

Previo a la puesta en servicio comercial de la línea, se ha procedido a un amolado preventivo de carril para mejorar las condiciones de circulación a Altas Velocidades, y reducir el ruido producido por la interacción ruedacarril que, a estas velocidades, es el predominante.

La línea Madrid-Sevilla es la primera línea férrea española electrificada con corriente monofásica de alta tensión y frecuencia industrial $(25.000$ voltios, $50 \mathrm{~Hz})$.

Los principales componentes de la electrificación son:

- Transporte de energía de la red de distribución a las subestaciones.

- Subestaciones de transformación, de 220.000 voltios a 25.000 voltios y salida hacia catenaria cada 40 kilómetros.

- Catenaria que transporte la energía (25.000 voltios a $50 \mathrm{~Hz}$, corriente alterna monofásica) desde las subestaciones a los pantógrafos.

Las características más importantes son:

- Gran uniformidad elástica, al disponer de péndola en Y.

- Postes de hormigón.

- Hilo de contacto de $129 \mathrm{~mm}^{2}$ de sección de cobre con un $0,1 \%$ de plata.

- Cable sustentador de bronce.

- Altura constante de 5,30 metros.

- Telemando de todos los equipos y teleseñalización de posición de aparatos y alarmas, que se integra en el telemando de instalaciones de seguridad.

Utiliza sistemas ya probados como son los enclavamientos electrónicos y la conducción automática de trenes, que permite prescindir de la señalización lateral, ya que la señalización del CAT en la cabina de conducción garantiza, en su totalidad, el flujo en la línea con las máximas garantías de seguridad.

Sus características principales son:

- Un enclavamiento electrónico cada 60 km (8 en total). 


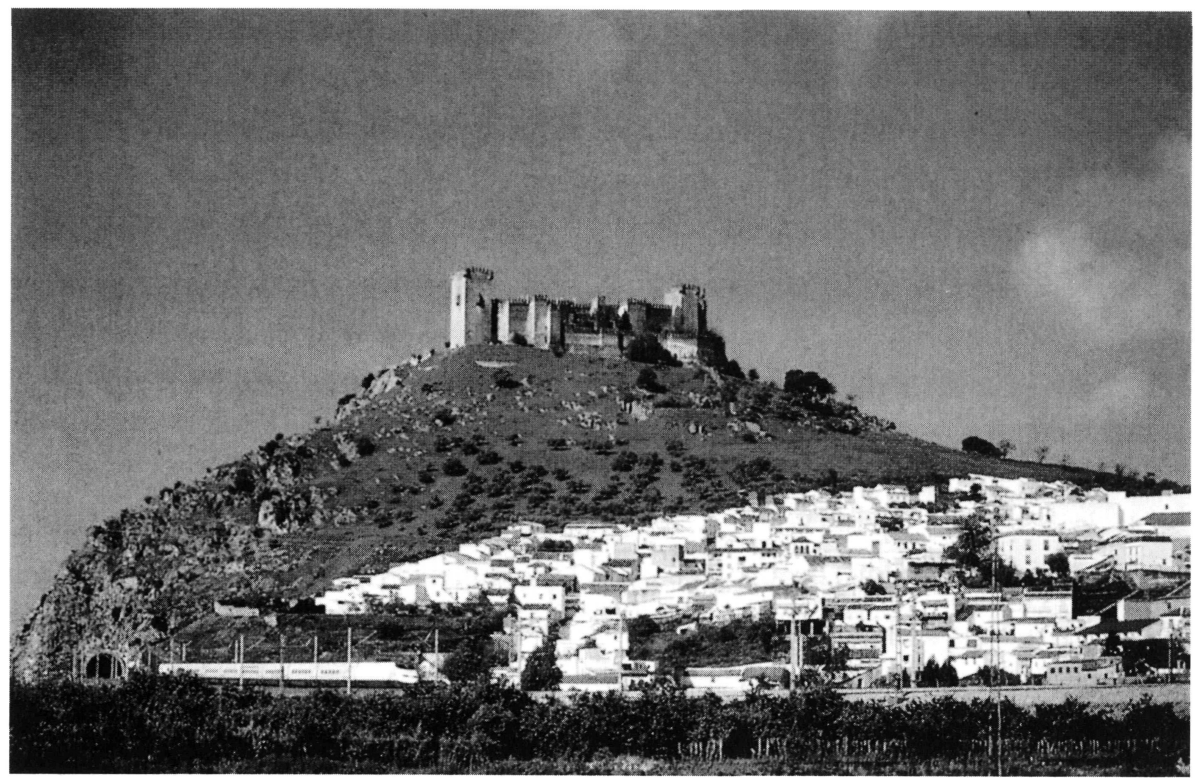

Túnel de Almodóvar del Río (Córdoba).

- Telemando desde un Puesto Central en MadridAtocha.

- Posibilidad de control descentralizado.

- Sistema de comunicación, redundante por disponibilidad integrado con gran capacidad y con máxima fiabilidad.

- Constitución por dos subsistemas:

- Correspondiente a las instalaciones de transmisión de comunicaciones y telemando.

- El de las instalaciones de supervisión de vigilancia e información.

- Sistema LZB de conducción automática que permita:

- Información y/o actuación en zonas de cambio.

- Elaboración de curvas de control de la velocidad en función de: longitud del tren, tipo de freno, velocidad máxima del tren y porcentaje de paso para cualquier tipo de circulación.

- Visión eléctrica de $10 \mathrm{~km}$ de vía.

- Equipos de supervisión, vigilancia e información de:

- Detección de cajas calientes.

- Vigilancia de bocas de túneles y pasos superiores.

- Alarmas contra incendios y antirrobo.

- Vigilancia por TV.

- Equipos cronométricos, indicadores de destino y megafonía.

(c) Consejo Superior de Investigaciones Científicas Licencia Creative Commons 3.0 España (by-nc)

\section{LA ATENCIÓN AL MEDIO AMBIENTE}

La nueva línea Madrid-Sevilla es la primera obra pública, de grandes magnitudes, que incorpora sistemáticamente medidas preventivas y correctoras de su impacto ambiental. Está vallada a ambos lados y no existe ningún paso a nivel, por lo que se han construido numerosos pasos superiores e inferiores (287 Ud.) para que no represente una barrera física. Además de la permeabilidad total que permiten los túneles y viaductos, existe un paso transversal cada $1,5 \mathrm{~km}$ de media, apto para personas, animales y vehículos. También se han construido pasos especialmente indicados para la fauna autóctona.

En terraplenes, vertederos y préstamos se han aplicado técnicas modernas de hidrosiembra con flora autóctona y remodelaciones, para su integración paisajística.

Las ventajas del ferrocarril, frente a otros medios de transporte, son:

- Ocupa relativamente poco espacio.

- Consume energía no contaminante.

- Nivel de ruido inferior al de transporte por carretera.

- Pocas posibilidades de agresión al entorno por parte de los usuarios. 


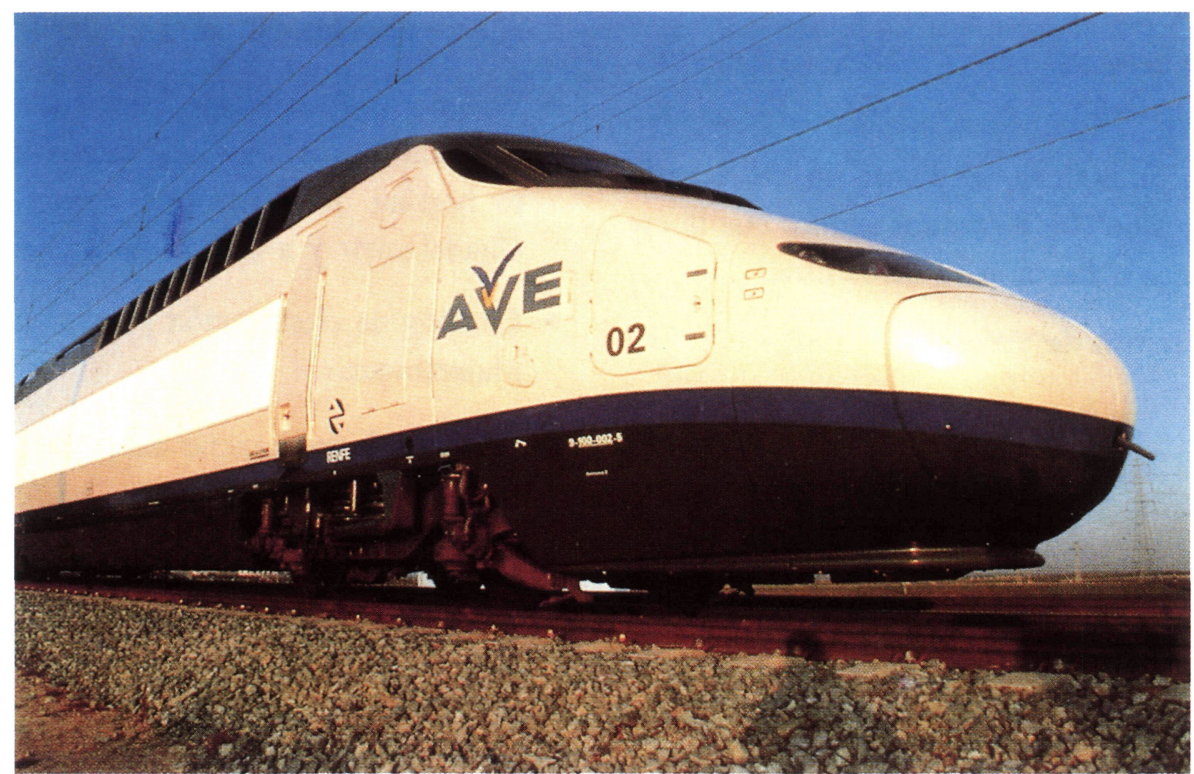

Tren español de Alta Velocidad.

Foto: MAN

- Bajo consumo energético:

- tres veces inferior al transporte por carretera,

- seis veces inferior al transporte aéreo.

Las medidas correctorạs del impacto ambiental ejecutadas son:

- Hidrosiembras y plantaciones a lo largo de la traza.

- Pantallas arbóreas.

- Plantaciones en préstamos y vertederos, preferentemente en zonas de interés ecológico y paisajístico.

- Restitución de riberas fluviales.

- Pasos de fauna.

Por su avanzado diseño, el tren español de Alta Velocidad, produce, a $250 \mathrm{~km} / \mathrm{h}$, un nivel sonoro similar al de un tren convencional a $160 \mathrm{~km} / \mathrm{h}$.

Para minimizar el nivel sonoro, se realizan:

- Pantallas antirruido en aquellas zonas, con trazado superficial, en el que la densidad y características de la población circundante así lo justifique.

- Variantes, como la de Ciudad Real, para evitar el paso por la ciudad.

(c) Consejo Superior de Investigaciones Científicas Licencia Creative Commons 3.0 España (by-nc)
- Soterramiento, como el de Córdoba, que anulan totalmente el impacto sonoro.

- Concentración de instalaciones en zonas menos pobladas de la ciudad, como Sevilla.

Las actuaciones urbanas de la nueva línea se centran en Madrid, Ciudad Real, Córdoba y Sevilla.

\section{MADRID}

- La línea Madrid-Sevilla comienza en la nueva Estación de Atocha que, junto con la estación de Cercanías y el intercambiador, constituyen un moderno complejo de transportes en la capital de España.

\section{CIUDAD REAL}

- Variante de doble vía de ancho internacional y vía única de ancho español, que elimina el paso del tren por la ciudad.

- Una nueva estación de viajeros, con vías de ancho internacional y ancho español.

- Una nueva estación de mercancías sobre la actual línea Manzanares-Ciudad Real, en las proximidades de Miguelturra.

http://informesdelaconstruccion.revistas.csic.es 
Informes de la Construcción, Vol. 44 n. ${ }^{\circ}$ 420, julio/agosto, 1992

\section{CÓRDOBA}

- Ampliación de la estación de clasificación de mercancías de "El Higuerón".

- Soterramiento de la línea, mediante un túnel artificial de 3,9 km, con vías de ambos anchos que libera grandes espacios urbanos para otros usos.

- Una nueva estación de viajeros.

\section{SEVILLA}

- Estación de Santa Justa, con los dos anchos de vía, que concentra los servicios de viajeros de la ciudad y libera los edificios de Plaza de Armas y San Bernardo.

- Soterramiento de 2,2 km de doble vía entre las esta- ciones de Santa Justa y La Salud, liberando terrenos que se transforman en avenidas urbanas.

- Variante norte a Huelva, que acorta en $6 \mathrm{~km}$ el trazado anterior y lo mejora. Esta obra suprime el efecto barrera del ferrocarril y abre la ciudad al río Guadalquivir y a la Cartuja. (Sede de la EXPO'92).

- Estación de clasificación y depósitos comerciales de Majarabique.

- Estación de mercancías de La Negrilla, que concentra este tipo de tráfico.

- Ramal directo desde Santa Justa al recinto de la EXPO'92, con apeadero ante una de sus principales entradas.

\section{publicaciones del ICCET/CSIC}

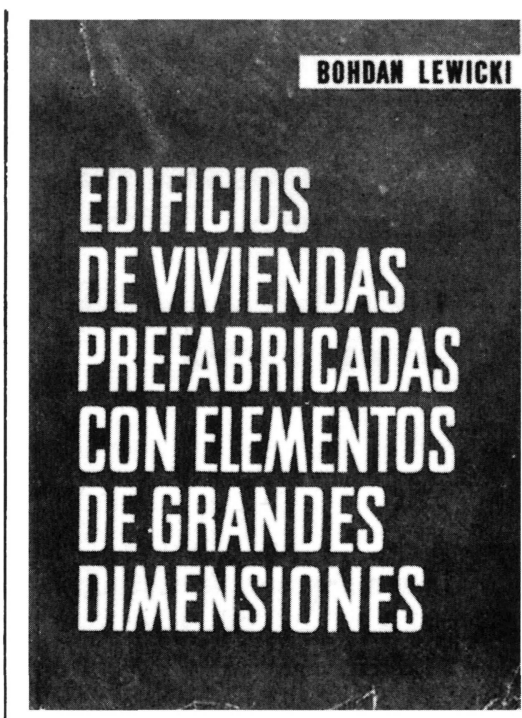

Bohdan Lewick

Este libro trata de los problemas relativos a la construcción de los edificios de viviendas o públicos realizados con elementos prefa. bricados de grandes dimensiones. Se han es. tudiado los problemas de arriostramiento, así como los que plantea la resistencia de los elementos y de la estructura; se han examinado las cuestiones de orden higrotér. mico, acústico y de resistencia al fuego; también se ha profundizado en el estudio de la estanquidad de los muros exteriores y de las juntas.

La obra incluye numerosas ilustraciones que dan detalles de diversas soluciones, asi como ejemplos de cálculo, tablas de valores numéricos, diagramas y ábacos.

Un volumen encuadernado en tela, de $24 \times 17 \mathrm{~cm}$, compuesto de 616 págs.

(c) Consejo Superior de Investigaciones Científicas Licencia Creative Commons 3.0 España (by-nc)
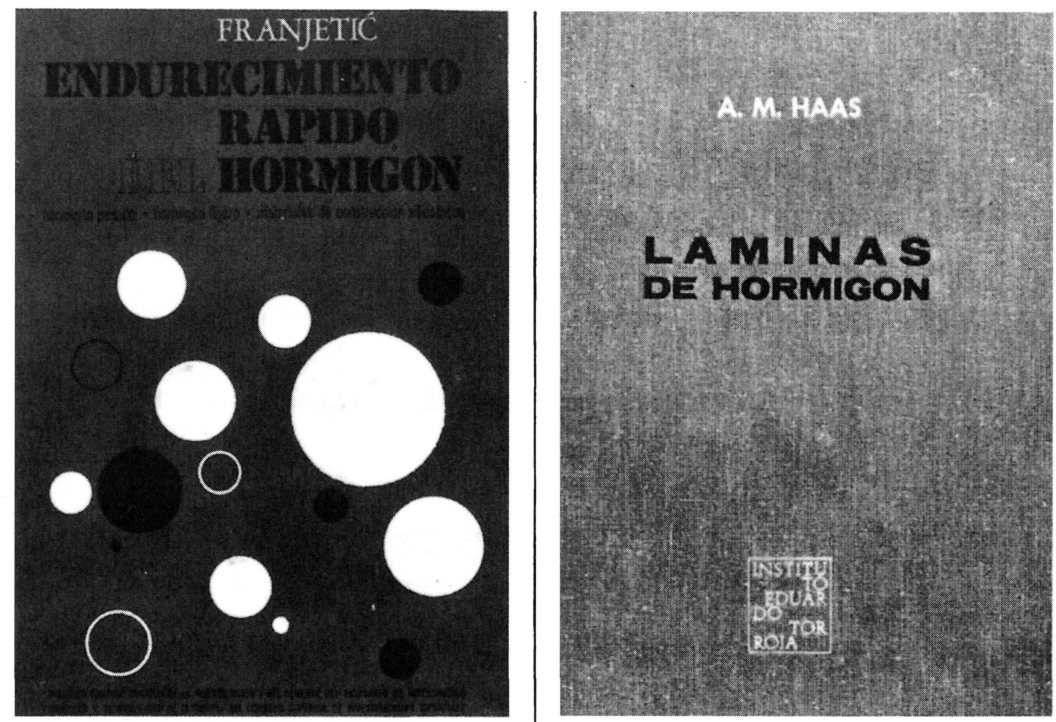

Zorislav Franjetić

En la obra de Franjetic se expone de una forma minuciosa, ordenada y sistemática, todo un cuerpo de doctrina que reúne el conocimiento actual sobre el endurecimiento nocimiento actual sobre el endurecimiento principios básicos y llega a las últimas consecuencias y realidades técnicas y eco. nómicas.

Es una obra de consulta, tanto para el in. vestigador sobre la materia, como para el proyectista y el realizador y montador de plantas e instalaciones y equipos de curado y endurecimiento rápido.

Un volumen encuadernado en cartóné, de $17 \times 24,5 \mathrm{~cm}$, compuesto de 385 págs. 110 figuras y 10 tablas.

\section{A. M. Haas}

Al escribir este libro el autor intentó poner a disposición de los estudiantes y de los ingenieros unos conocimientos prácticos, adecuados para servir de guía en el diseño y construcción de láminas delgadas de hor. migón.

El autor está convencido de que el éxito en el diseño de una lámina exige, por parte del proyectista, un examen de las tres fases por las que pasa la materialización de la lámina: el diseño, el análisis estructural y la construcción de la estructura.

Un volumen encuadernado en tela, de $17 \times 24,5 \mathrm{~cm}$, compuesto de 420 págs., 141 figuras, 22 fotografías y 6 tablas. 